\title{
Half-sided Modular Inclusion and the Construction of the Poincaré Group
}

\section{H.J. Borchers}

Institut für Theoretische Physik der Universität Göttingen, Bunsenstrasse 9,

D-37073 Göttingen, Germany. E-mail: Borchers@Theorie.Physik.UNI-Goettingen.DE

Received: 20 December 1995 /Accepted: 26 January 1996

\begin{abstract}
In this note we will give a construction of the Poincaré group out of the modular groups of the wedge algebras provided the groups act on the algebra of every double cone like the associated Lorentz boosts. This construction will use the concept of half-sided modular inclusions instead of the first and second cohomology of the Poincaré group as used by Brunetti, Guido and Longo. By our method we obtain directly the Poincaré group and not its covering group.
\end{abstract}

\section{Introduction}

The modular groups of different subalgebras appearing in the theory of local observables have been used to construct the symmetry-group of the whole theory. There are two examples, namely the construction of the Möbius-group in chiral quantum field theory by H.-W. Wiesbrock [Wie2] and the construction of the Poincare group in the four-dimensional theory of local observables due to Brunetti, Guido and Longo [BGL] and of Guido and Longo [GL].

If one starts from two or more one-parametric groups, these groups will generally create an infinite dimensional group. In the above-mentioned examples, however, we obtain only a finite dimensional Lie-group and one is interested to understand the reason for this reduction. By superficial consideration one might think that the reduction is due to the locality of the action of the modular groups we are starting with. These locality assumptions are consequences of either the result of Bisognano and Wichmann [BW1,2] or of the conclusions of Borchers [Bch1]. But this alone does not lead to a reduction and I think that this reduction is due to the fact that there are pairs of algebras the intersection of which fulfills the condition of half-sided modular inclusion with respect to both algebras.

With two algebras fulfilling the condition of half-sided modular inclusion one finds a group $U(t)$ which maps the large algebra onto the small one if $t$ takes the value 1 . This group has either a positive or a negative spectrum, from which one is usually able to show the uniqueness of this group. This uniqueness seems to be the 
reason why one obtains finite dimensional Lie-groups and not a central extension of them.

Probably it will be extremely difficult to prove such a conjecture, because the modular groups of two algebras will immediately generate an infinity of algebras and it is almost impossible to classify their intersections. Therefore, one can only look for examples in order to support the presumption. In the case of the chiral field theory it is easy to see that the concept of half-sided modular inclusion, introduced by Wiesbrock [Wie1], is the source of the reduction. In this note we will treat the case of Brunetti, Guido and Longo and give a construction of the Poincare group (and not of its covering group) by using half-sided modular inclusions instead of cohomology theory. Also here we start from the assumption that the modular groups of the wedge algebras act like their associated groups of Lorentz boosts. The assumed locality of the modular action will be used in order to construct subalgebras fulfilling the condition of half-sided modular inclusion with respect to two or more wedge algebras.

We start from a representation of a quantum field theory of local observables with similar properties as in the vacuum sector. For a given domain $O$ the von Neumannalgebra, generated by the representation of the local observables located in $O$, will be denoted by $\mathscr{M}(O)$. The cyclic and separating vector for these algebras will always be the same vector $\Omega$. The starting point of the investigation are the wedges. They are defined with help of two linearly independent lightlike vectors $\ell_{1}, \ell_{2}$ belonging to the forward lightcone by the formula

$$
W\left[\ell_{1}, \ell_{2}\right]=\left\{\alpha \ell_{1}+\beta \ell_{2}+\ell^{\perp} ; \quad \alpha>0, \beta<0,\left(\ell^{\perp}, \ell_{i}\right)=0, i=1,2\right\},
$$

the scalar product means the Minkowski-space product. The translated wedges will be denoted by $W\left[\ell_{1}, \ell_{2}, a\right]$. They will be needed only for the construction of the translations. It is often convenient to choose two vectors $\ell_{3}, \ell_{4}$ in the space perpendicular to $\ell_{1}$ and $\ell_{2}$ spanning this space. For $\ell_{3}, \ell_{4}$ we require the conditions

$$
\left(\ell_{1}, \ell_{3}\right)=\left(\ell_{2}, \ell_{3}\right)=\left(\ell_{1}, \ell_{4}\right)=\left(\ell_{2}, \ell_{4}\right)=\left(\ell_{3}, \ell_{4}\right)=0, \quad \ell_{3}^{2}=\ell_{4}^{2}=-1 .
$$

For many calculations it is convenient to introduce a fixed time-direction $t, t^{2}=1$ in the forward lightcone and a fixed coordinate system. This is equivalent to introduce a second, euclidean, metric on the Minkowski-space. The scalar product in this metric will be denoted by angular brackets $\langle.,$.$\rangle . If \ell_{1}, \ldots, \ell_{4}$ is a basis of the Minkowskispace then we denote the dual basis with respect to the euclidean product by $m_{i}$, i.e.,

$$
\left\langle\ell_{i}, m_{j}\right\rangle=\delta_{i, j}, \quad i, j=1, \ldots, 4 .
$$

In this setting the Lorentz boosts belonging to the wedge $W\left[\ell_{1}, \ell_{2}\right]$ have the following representation:

$$
\begin{gathered}
\left.\Lambda\left[\ell_{1}, \ell_{2}\right](t)=\ell_{1}\right\rangle \mathrm{e}(t)\left\langle m_{1}+\ell_{2}\right\rangle \mathrm{e}(-t)\left\langle m_{2}+\ell_{3}\right\rangle\left\langle m_{3}+\ell_{4}\right\rangle\left\langle m_{4}\right. \\
\text { with } \mathrm{e}(t)=\mathrm{e}^{-2 \pi t} .
\end{gathered}
$$

Our assumptions are the same as those of Brunetti,Guido and Longo, namely, that the modular group belonging to a wedge algebra acts on the algebra associated with any double cone in the same manner as the associated Lorentz boosts.

$$
\operatorname{ad} \Delta\left[\ell_{1}, \ell_{2}, a\right]^{\mathrm{i} t} \mathscr{M}(O)=\mathscr{M}\left(\Lambda\left[\ell_{1}, \ell_{2}, a\right](t) O\right) .
$$


This implies for the algebra of the wedge the relation

$$
\operatorname{ad} \Delta\left[\ell, \ell^{\prime}, b\right]^{\mathrm{i} s} \Delta\left[\ell_{1}, \ell_{2}, a\right]^{\mathrm{i} t}=\Delta\left[\Lambda\left[\ell, \ell^{\prime}, b\right](s) \ell_{1}, \Lambda\left[\ell, \ell^{\prime}, b\right](s) \ell_{2}, \Lambda\left[\ell, \ell^{\prime}, b\right](s) a\right]^{\mathrm{it}} .
$$

Formula (1.6) is an important ingredient which will often be used.

In the next section we will collect some known results which are needed for our investigations. Furthermore, we describe pairs of algebras in the theory of local observables fulfilling the condition of half-sided modular inclusion. In Sect. 3 we construct the translations and in the last section the Lorentz group will be constructed.

For the unit operator acting on the representation Hilbert space we use the symbol 11. The unit matrix acting on $\mathbb{R}^{4}$ is denoted by 1 .

\section{On Pairs of Algebras Fulfilling the Condition of Half-sided Modular Inclusion}

First we want to collect some known results.

Let $\mathscr{M}$ be a von Neumann algebra with cyclic and separating vector $\Omega$ then the modular operator and the modular conjugation of the pair $(\mathscr{\mathscr { C }}, \Omega)$ will be denoted by $\Delta_{\mathscr{H}}$ and $J_{\mathscr{M}}$, respectively. The combination $J_{\mathscr{C}} \Delta_{\mathscr{M}}^{1 / 2}=S_{\mathscr{H}}$ will be called the Tomita conjugation of $(\mathscr{C}, \Omega)$. Since in all our investigations the vector $\Omega$ will be kept fixed we drop this vector in the characterization of the appearing objects. The essential ingredient of our investigation is the following result which we quote for the convenience of the reader:

Theorem 2.1 (Borchers [Bch1], Wiesbrock [Wie1,2]). Let $\mathscr{M b}$ be a von Neumann algebra with cyclic and separating vector $\Omega$.

(1) If $U(t)$ is a continuous one-parametric unitary group with

i. $U(t) \Omega=\Omega$,

ii. $U(t)$ has a non-negative generator,

iii. $\operatorname{ad} U(t) \mathscr{M} \subset \mathscr{M}$ for $t \geq 0$,

then with $\mathscr{N}_{t}:=\operatorname{ad} U(t) \mathscr{M}$ follows:

$$
\operatorname{ad} \Delta_{\mathscr{M}}^{\mathrm{it}} \mathscr{N}_{s}=\mathscr{N}_{\mathrm{e}^{-2 \pi t_{s}}} \text { and ad } J_{\mathscr{M}} \mathscr{N}_{s}=\left(\mathscr{N}_{-s}\right)^{\prime}
$$

(2) If $\mathscr{N}$ is a sub von Neumann algebra of $\mathscr{M}$ having $\Omega$ as cyclic vector and

$$
\text { ad } \Delta_{\mathscr{M}}^{\mathrm{it}} \mathscr{N} \subset \mathscr{N} \text { for } t \leq 0,
$$

then a continuous unitary group $U(t)$ exists fulfilling the conditions of (1) and

$$
\mathscr{N}=\operatorname{ad} U(1) \cdot \mathscr{C} \text {. }
$$

(3) If (1) or equivalently (2) is fulfilled then the following relations hold between the different operators:

$$
\begin{aligned}
\operatorname{ad} \Delta_{\mathscr{C}}^{\mathrm{i} t} U(s) & =U\left(\mathrm{e}^{-2 \pi t} s\right), \\
\operatorname{ad} J_{\mathscr{H}} U(s) & =U(-s), \\
U\left(\mathrm{e}^{-2 \pi t}-1\right) & =\Delta_{\mathscr{M}}^{\mathrm{i} t} \Delta_{\mathscr{N}}^{\mathrm{i} t} \\
U(-2) & =J_{\mathscr{C}} J_{\mathscr{N}} .
\end{aligned}
$$

Remarks 2.2. 
(1) The property $\mathscr{N} \subset \mathscr{M}$ and ad $\Delta_{\mathscr{C}}^{\text {it }} \mathscr{N} \subset \mathscr{N}$ for $t \leq 0$ is called -half-sided modular inclusion.

(2) If one abbreviates ad $U(t) \mathscr{M}$ by $\mathscr{N}_{t}$ then one gets the inclusions

$$
\begin{gathered}
\mathscr{N}_{t} \subset \mathscr{M} \text { for } t \geq 0, \\
\mathscr{N}_{t} \supset \mathscr{M}_{6} \text { for } t \leq 0, \\
\mathscr{N}_{t_{1}} \subset \mathscr{N}_{t_{2}} \text { for } t_{1} \geq t_{2} .
\end{gathered}
$$

Concerning the uniqueness of the group $U(t)$ the following result is known:

Theorem 2.3 [Bch2,Prop. 3.4]. Let $\mathscr{M}_{a}$ and $\mathscr{N}_{a}, a \in \mathbb{R}$ be two families of von Neumann algebras on the Hilbert spaces $\mathscr{H}_{m}, \mathscr{H}_{n}$ with the cyclic and separating vector $\Omega_{m}, \Omega_{n}$, respectively. Assume there are continuous unitary one parametric groups $U_{\mathscr{M}}(a), U_{\mathscr{N}}(a)$ both fulfilling spectrum condition and define

$$
\mathscr{M}_{a}=U_{\mathscr{L}}(a) \mathscr{M}_{0} U_{\mathscr{C}}(-a), \quad \mathscr{N}_{a}=U_{\mathscr{N}}(a) \mathscr{N}_{0} U_{\mathscr{N}}(-a) .
$$

Assume moreover

$$
\mathscr{M}_{a} \subset \mathscr{M}_{b}, \quad \mathscr{N}_{a} \subset \mathscr{N}_{b} \text { for } a>b .
$$

If there exists a unitary map $W$ with $W \mathscr{H}_{n}=\mathscr{H}_{m}$ and $W \Omega_{n}=\Omega_{m}$ and in addition

$$
\mathscr{C}_{0}=W \mathscr{N}_{0} W^{*}, \text { and } \mathscr{\mathscr { C }}_{1}=W \mathscr{N}_{1} W^{*} \text {, }
$$

then follows

$$
\begin{aligned}
\mathscr{M}_{a} & =W \mathscr{N}_{a} W^{*} \quad \forall a \in \mathbb{R}, \\
U_{m}(a) & =W U_{n}(a) W^{*} .
\end{aligned}
$$

The same is true if we require that $\mathscr{M}_{0}$ and $\mathscr{M}_{1}$ as well as $\mathscr{N}_{0}$ and $\mathscr{N}_{1}$ both fulfill modular inclusion for negative arguments of the modular groups.

Remark 2.4.

(1) If $\mathscr{N}$ fulfills condition (2) of Theorem 2.1 then $\operatorname{ad} U(a) \mathscr{N}=$ ad $U(a+1) \mathscr{M}=\mathscr{N}_{a+1}$ fulfills the same condition for $a>0$. The corresponding group $U_{\mathscr{N}_{a}}(t)$ is obtained by scaling

$$
U_{\mathscr{V}_{a}}(t)=U(a t) .
$$

(2) If $\mathscr{N}$ fulfills condition (2) of Theorem 2.1 then $\Delta_{\mathscr{N}}$ and $J_{\mathscr{N}}$ can be computed with help of $\Delta_{\mathscr{C}}$ and $U(1)$ or with help of $J_{\mathscr{H}}$ and $U(1)$, respectively. Hence one finds the same relations

$$
\begin{aligned}
\operatorname{ad} \Delta_{\mathscr{V}}^{\mathrm{i} t} U(s) & =U\left(\mathrm{e}^{-2 \pi t} s\right), \\
\operatorname{ad} J_{\mathscr{N}} U(s) & =U(-s) .
\end{aligned}
$$

Next we turn to the characterization of pairs of algebras fulfilling the condition of half-sided modular inclusion. For this investigation it is important that the modular groups of the wedge algebras act like the associated Lorentz groups.

Lemma 2.5. Let $\ell, \ell^{\prime}$ be two linear independent lightlike vectors in the boundary of the forward light cone, then the pair

$$
\mathscr{M}\left(W\left[\ell, \ell^{\prime}, a\right]\right) \supset \mathscr{M}\left(W\left[\ell, \ell^{\prime}, a+\lambda \ell\right]\right), \quad \lambda>0
$$


fulfills the condition of -half-sided modular inclusion. Correspondingly the pair

$$
\mathscr{M}\left(W\left[\ell, \ell^{\prime}, a\right]\right) \supset \mathscr{M}\left(W\left[\ell, \ell^{\prime}, a-\mu \ell^{\prime}\right]\right), \quad \mu>0
$$

fulfills the condition of thalf-sided modular inclusion.

Proof. For the wedge $W\left[\ell, \ell^{\prime}, 0\right]$ with $\ell=(1,1,0,0), \ell^{\prime}=(1,-1,0,0)$ the Lorentz boosts associated with the modular group of the wedge algebra are of the form

$$
\Lambda(t)=\left(\begin{array}{cccc}
\cosh 2 \pi t & -\sinh 2 \pi t & 0 & 0 \\
-\sinh 2 \pi t & \cosh 2 \pi t & 0 & 0 \\
0 & 0 & 1 & 0 \\
0 & 0 & 0 & 1
\end{array}\right)
$$

From this follows (2.1) and (2.2) in case $a=0$ and $\ell, \ell^{\prime}$ are the above vectors. The general statement is obtained by applying the corresponding Poincaré transformation (as coordinate transformation) to the special situation.

The first step in our further construction will be to look at the family of wedges having one light ray in common,

$$
\left\{W\left[\ell, \ell_{2}\right] ; \ell \text { fixed }\right\},
$$

and at the stabilizer group of the vector $\ell$. It is well known that the stabilizer $S(\ell)$ of a lightlike vector is isomorphic to the euclidean transformation of $\mathbb{R}^{2}$. (See e.g. Gelfand, Minlos and Shapiro [GMS].) The rotations are the transformations around the space-direction of the light ray. In order to understand the translations let us introduce a second lightlike vector $\ell_{2}$ which we choose in such a way that $\ell, t, \ell_{2}$ lie in one two-plane. Let $T(\ell)$ be the tangent hyperplane at the forward lightcone $V^{+}$containing the vector $\ell$. Then the affine hyperplane $\ell_{2}+T(\ell)$ intersects $\partial V^{+}$in a two-dimensional set (parabola) homeomorphic to $\mathbb{R}^{2}$. The translations of $S(\ell)$ have this set as orbit.

In the concrete example

$$
\ell=(1,1,0,0), \ell_{2}=(1,-1,0,0), \ell_{3}=(0,0,1,0), \ell_{4}=(0,0,0,1)
$$

these translations become $\left(a=\left(a_{1}, a_{2}\right) \in \mathbb{R}^{2}\right)$

$$
\Lambda^{\ell}(a)=\left(\begin{array}{cccc}
1+\frac{a^{2}}{2} & -\frac{a^{2}}{2} & a_{1} & a_{2} \\
\frac{a^{2}}{2} & 1-\frac{a^{2}}{2} & a_{1} & a_{2} \\
a_{1} & -a_{1} & 1 & 0 \\
a_{2} & -a_{2} & 0 & 1
\end{array}\right) .
$$

(See also R. Jost [Jo] Appendix.) It is easy to check that this is a representation of the two-dimensional translation group,

$$
\Lambda^{\ell}(a) \Lambda^{\ell}(b)=\Lambda^{\ell}(a+b) .
$$

The transformed vectors $\ell_{i}(a):=\Lambda^{\ell_{1}}(a) \ell_{i}$ are of the form

$$
\begin{aligned}
\ell(a) & =\ell \\
\ell_{2}(a) & =a^{2} \ell+\ell_{2}+2 a_{1} \ell_{3}+2 a_{2} \ell_{4}=\left(1+a^{2},-1+a^{2}, 2 a_{1}, 2 a_{2}\right), \\
\ell_{3}(a) & =a_{1} \ell+\ell_{3}=\left(a_{1}, a_{1}, 1,0\right) \\
\ell_{4}(a) & =a_{2} \ell+\ell_{4}=\left(a_{2}, a_{2}, 0,1\right)
\end{aligned}
$$


The dual base can easily be computed and one finds

$$
\begin{aligned}
& m_{1}(a)=\frac{1}{2} \ell+\frac{a^{2}}{2} \ell_{2}-a_{1} \ell_{3}-a_{2} \ell_{4}, \\
& m_{2}(a)=\frac{1}{2} \ell_{2}, \\
& m_{3}(a)=-a_{1} \ell_{2}+\ell_{3}, \\
& m_{4}(a)=-a_{2} \ell_{2}+\ell_{4} .
\end{aligned}
$$

Using the representation (2.6) and (2.6') we list the euclidean scalar products for later use,

$$
\begin{aligned}
& \left\langle m_{1}(a), \ell\right\rangle=1, \quad\left\langle m_{2}(a), \ell\right\rangle=0, \\
& \left\langle m_{1}(a), \ell_{2}(b)\right\rangle=(b-a)^{2}, \quad\left\langle m_{2}(a), \ell_{2}(b)\right\rangle=1 \text {, } \\
& \left\langle m_{1}(a), \ell_{3}(b)\right\rangle=b_{1}-a_{1}, \quad\left\langle m_{2}(a), \ell_{3}(b)\right\rangle=0 \text {, } \\
& \left\langle m_{1}(a), \ell_{4}(b)\right\rangle=b_{2}-a_{2}, \quad\left\langle m_{2}(a), \ell_{4}(b)\right\rangle=0 \text {, } \\
& \left\langle m_{3}(a), \ell\right\rangle=0, \quad\left\langle m_{4}(a), \ell\right\rangle=0, \\
& \left\langle m_{3}(a), \ell_{2}(b)\right\rangle=2\left(b_{1}-a_{1}\right), \quad\left\langle m_{4}(a), \ell_{2}(b)\right\rangle=2\left(b_{2}-a_{2}\right) \text {, } \\
& \left\langle m_{3}(a), \ell_{3}(b)\right\rangle=1, \quad\left\langle m_{4}(a), \ell_{3}(b)\right\rangle=0 \text {, } \\
& \left\langle m_{3}(a), \ell_{4}(b)\right\rangle=0, \quad\left\langle m_{4}(a), \ell_{4}(b)\right\rangle=1 .
\end{aligned}
$$

Using the two basis $\ell$ and $m$ we get for $\Lambda^{\ell}(a)$ the representation $\left(m_{i}=m_{i}(0)\right)$

$$
\begin{aligned}
& \left.\Lambda^{\ell}(a)=\ell\right\rangle\left\langle m_{1}+\ell_{2}(a)\right\rangle\left\langle m_{2}+\ell_{3}(a)\right\rangle\left\langle m_{3}+\ell_{4}(a)\right\rangle\left\langle m_{4}=\right. \\
& \quad \ell\rangle\left\langle m_{1}(b)+\ell_{2}(a+b)\right\rangle\left\langle m_{2}(b)+\ell_{3}(a+b)\right\rangle\left\langle m_{3}(b)+\ell_{4}(a+b)\right\rangle\left\langle m_{4}(b),\right.
\end{aligned}
$$

and for $\Lambda\left[\ell, \ell_{2}(a)\right](t)$ the representation

$$
\left.\Lambda\left[\ell, \ell_{2}(a)\right](t)=\ell\right\rangle \mathrm{e}(t)\left\langle m_{1}(a)+\ell_{2}(a)\right\rangle \mathrm{e}(-t)\left\langle m_{2}(a)+\ell_{3}(a)\right\rangle\left\langle m_{3}(a)+\ell_{4}(a)\right\rangle\left\langle m_{4}(a) .\right.
$$

In order to relate $\Lambda^{\ell}(a)$ with the boosts $\Lambda\left[\ell, \ell_{2}\right](t)$ we compute the following product with help of (2.3') and (2.6”):

$$
\begin{aligned}
\Lambda\left[\ell, \ell_{2}(a)\right](t) & \left.\Lambda\left[\ell, \ell_{2}(b)\right](-t)=\ell\right\rangle\left\langle m_{1}(b)+\ell_{2}(a)\right\rangle\left\langle m_{2}(b)+\ell_{3}(a)\right\rangle\left\langle m_{3}(b)\right. \\
\left.+\ell_{4}(a)\right\rangle\left\langle m_{4}(b)\right. & +\mathrm{e}(t)\{\ell\rangle\left\langle m_{1}(a), \ell_{3}(b)\right\rangle\left\langle m_{3}(b)+\ell\right\rangle\left\langle m_{1}(a), \ell_{4}(b)\right\rangle\left\langle m_{4}(b)\right. \\
& \left.+\ell_{3}(a)\right\rangle\left\langle m_{3}(a), \ell_{2}(b)\right\rangle\left\langle m_{2}(b)+\ell_{4}(a)\right\rangle\left\langle m_{4}(a), l_{2}(b)\right\rangle\langle m 2(b)\} \\
& +\mathrm{e}(t)^{2}\{\ell\rangle\left\langle m_{1}(a), \ell_{2}(b)\right\rangle\left\langle m_{2}(b)\right\} .
\end{aligned}
$$

Comparing this result with the second equation of (2.5') we get

$$
\Lambda^{\ell}(a-b)=\lim _{t \rightarrow \infty} \Lambda\left[\ell, \ell_{2}(a)\right](t) \Lambda\left[\ell, \ell_{2}(b)\right](-t) .
$$

Later we will have to show that the corresponding product $\Delta\left[\ell_{1}, \ell_{2}(a)\right]^{i t} \times$ $\Delta\left[\ell_{1}, \ell_{2}(b)\right]^{-\mathrm{i} t}$ converges for $t \rightarrow \infty$ strongly to a unitary operator $U(a, b)$ and that this operator acts local on every double cone, i.e.,

$$
\operatorname{ad} U(a, 0) \mathscr{M}(O)=\mathscr{M}\left(\Lambda^{\ell}(a) O\right) .
$$


This will be done in Sect. 4 .

Now we are prepared to look at the inersection of two wedges with a common lightlike vector. We show

Lemma 2.6. The algebra $\mathscr{M}\left(W\left[\ell, \ell_{1}\right] \cap W\left[\ell, \ell_{2}\right]\right)$ fulfills the condition of - halfsided modular inclusion with respect to both algebras $\mathscr{M}\left(W\left[\ell, \ell_{1}\right]\right)$ and $\mathscr{M}\left(W\left[\ell, \ell_{2}\right]\right)$.

Proof. We identify $\ell_{1}$ with $\ell_{2}(a)$ and $\ell_{2}$ with $\ell_{2}(b)$. The wedge $W\left[\ell, \ell_{2}(a)\right]$ has the representation

$$
W\left[\ell, \ell_{2}(a)\right]=\left\{\alpha \ell+\beta \ell_{2}(a)+\gamma_{1} \ell_{3}(a)+\gamma_{2} \ell_{4}(a) ; \alpha>0, \beta<0, \gamma_{1}, \gamma_{2} \in \mathbb{R}\right\} .
$$

We apply to this expression the identity in the form of

$$
1=\ell\rangle\left\langle m_{1}(b)+\ell_{2}(b)\right\rangle\left\langle m_{2}(b)+\ell_{3}(b)\right\rangle\left\langle m_{3}(b)+\ell_{4}(b)\right\rangle\left\langle m_{4}(b),\right.
$$

and obtain with help of (2.6")

$$
\begin{aligned}
W\left[\ell, \ell_{2}(a)\right] & \left.\left.=\{\ell\rangle\left[\alpha+\beta(a-b)^{2}+\langle\gamma, a-b\rangle\right]+\ell_{2}(b)\right\rangle \beta+\ell_{3}(b)\right\rangle\left[2\left(a_{1}-b_{1}\right)+\gamma_{1}\right] \\
& \left.\left.+\ell_{4}(b)\right\rangle\left[2\left(a_{2}-b_{2}\right)+\gamma_{2}\right] ; r \alpha>0, \beta<0, \gamma_{1}, \gamma_{2} \in \mathbb{R}\right\} .
\end{aligned}
$$

Therefore, the intersection $W\left[\ell, \ell_{1}\right] \cap W\left[\ell, \ell_{2}\right]$ is characterized by

$$
\begin{aligned}
&\left\{\alpha \ell+\beta \ell_{2}(a)+\gamma_{1} \ell_{3}(a)+\gamma_{2} \ell_{4}(a)\right. ; \alpha>0, \beta<0, \gamma_{1}, \gamma_{2} \in \mathbb{R}, \\
&\left.\left\{\alpha+\beta(a-b)^{2}+\langle\gamma, a-b\rangle\right\}>0\right\} .
\end{aligned}
$$

Applying $\Lambda\left[\ell, \ell_{2}(a)\right](t)$ to (2.9) amounts to replace the coefficient $\alpha$ of $\ell$ by e $(t) \ell$ and the coefficient $\beta$ of $\ell_{2}(a)$ by $\mathrm{e}(-t) \beta$. Hence applying $\Lambda\left[\ell, \ell_{2}(a)\right](t)$ to points of the intersection leads to points

$$
\begin{gathered}
\left\{\mathrm{e}(t) \alpha \ell+\mathrm{e}(-t) \beta \ell_{2}(a)+\gamma_{1} \ell_{3}(a)+\gamma_{2} \ell_{4}(a)\right. \\
\left.\alpha>0, \beta<0, \gamma_{1}, \gamma_{2} \in \mathbb{R},\left(\alpha+\beta(a-b)^{2}+\langle\gamma, a-b\rangle\right)>0\right\} .
\end{gathered}
$$

Since we can write

$$
\begin{aligned}
\mathrm{e}(t) \alpha+\mathrm{e}(-t) \beta(a-b)^{2}+\langle\gamma, a-b\rangle & =\alpha+\beta(a-b)^{2}+\langle\gamma, a-b\rangle+(\mathrm{e}(t)-1) \alpha \\
& -(1-\mathrm{e}(-t)) \beta(a-b)^{2}
\end{aligned}
$$

we find that the intersection is mapped into the intersection for $\mathrm{e}(t) \geq 1$ which means for $t \leq 0$. Since the modular groups of the wedge algebras act as their associated Lorentz boosts we conclude that the algebra $\mathscr{M}\left(W\left[\ell, \ell_{2}(a)\right] \cap W\left[\ell, \ell_{2}(b)\right]\right)$ fulfills the condition of - half-sided modular inclusion with respect to the algebra $\mathscr{M}\left(W\left[\ell, \ell_{2}(a)\right]\right)$ and by symmetry also with respect to the algebra $\mathscr{C}\left(W\left[\ell, \ell_{2}(b)\right]\right)$.

Remark 2.7. If we look at three wedges with one common lightlike vector $\ell$, i.e. $W\left[\ell, \ell_{1}\right], W\left[\ell, \ell_{2}\right], W\left[\ell, \ell_{3}\right]$ then the algebra of the intersection

$$
\mathscr{M}\left(W\left[\ell, \ell_{1}\right] \cap W\left[\ell, \ell_{2}\right] \cap W\left[\ell, \ell_{3}\right]\right)
$$

also fulfills the condition of -half-sided modular inclusion with respect to all three algebras $W\left[\ell, \ell_{i}\right], i=1,2,3$. This is a consequence of the identity 


$$
\begin{aligned}
\mathscr{M}\left(W\left[\ell, \ell_{1}\right] \cap W\left[\ell, \ell_{2}\right] \cap W\left[\ell, \ell_{3}\right]\right) \\
=\mathscr{M}\left(W [ \ell , \ell _ { 1 } ] \cap W \left[\ell, \ell_{2}[) \cap \mathscr{M}\left(W\left[\ell, \ell_{1}\right] \cap W\left[\ell, \ell_{3}\right]\right)\right.\right.
\end{aligned}
$$

and the fact that both algebras on the right-hand side fulfill the condition of - halfsided modular inclusion with respect to $\mathscr{M}\left(W\left[\ell, \ell_{1}\right]\right)$ and hence also for the intersection. For the other two algebras the statement follows by symmetry.

\section{Construction of the Translations}

We start our investigation by looking at the family of wedges $W\left[\ell, \ell^{\prime}, a\right]$ where $\ell$ and $\ell^{\prime}$ are fixed and $a$ is of the form $a=\lambda \ell+\mu \ell^{\prime}$. Therefore, we suppress in the first part the indices $\left[\ell, \ell^{\prime}\right]$ and write simply $W[a], \Delta[a]^{\mathrm{i} t}$, and so on.

Let $W[a]$ and $W[a+\lambda \ell]$ be two wedges and $\lambda>0$. Then by Lemma 2.5 the algebra $\mathscr{M}(W[a+\lambda \ell])$ fulfills the condition of - half-sided modular inclusion with respect to the algebra $\mathscr{M}(W[a])$. Hence by Theorem 2.1 a unitary group $U[a, \lambda \ell](t)$ exists with positive generator fulfilling

$$
\operatorname{ad} U[a, \lambda \ell](1) \mathscr{M}(W[a])=\mathscr{M}(W[a+\lambda \ell]) .
$$

Furthermore, this group satisfies the following properties $\left(\mathrm{e}(t)=\mathrm{e}^{-2 \pi t}\right)$ :

$$
\begin{aligned}
U[a, \lambda \ell](t) \Omega & =\Omega, \\
\operatorname{ad} \Delta[a]^{\mathrm{i}} U[a, \lambda \ell](s) & =U[a, \lambda \ell](\mathrm{e}(t) s), \\
\operatorname{ad} U[a, \lambda \ell](s) \mathscr{U}(W[a]) & =\mathscr{U}(W[a+s \lambda \ell]), \\
U[a, \lambda \ell](1-\mathrm{e}(t)) & =\Delta[a+\lambda \ell]^{\mathrm{i} t} \Delta[a]^{-\mathrm{i} t} .
\end{aligned}
$$

For the first two lines see Theorem 2.1. The second line together with (1.6) implies the third line. The last line follows from (3.1) and from the second line of (3.2). Because of Theorem 2.3 the group $U[a, \lambda \ell](s)$ is uniquely defined by the properties listed in the first and third line together with the positivity of the spectrum. From the last line of (3.2) we obtain

$$
U[a, \lambda \ell](1)=\lim _{t \rightarrow \infty} \Delta[a+\lambda \ell]^{\mathrm{i} t} \Delta[a]^{-\mathrm{i} t} .
$$

Notice that by the last line of (3.2) the limit converges in the weak and hence in the strong topology. Moreover, from representation (3.3) we see that $U[a, \lambda \ell](s)$ acts like the translation in the $\ell$ direction. Hence by the uniqueness Theorem 2.3 we find that this is independent of $a$, i.e.

$$
U[a, \lambda \ell](t)=U[b, \lambda \ell](t) .
$$

The mentioned uniqueness of the groups $U[a, \lambda \ell](t)$ implies for $\lambda, \mu \neq 0$ the identity

$$
U[a, \lambda \ell](s)=U[a, \mu \ell]\left(\frac{\lambda}{\mu} s\right) .
$$

Hence we only have to deal with groups $U[\ell](s)$.

Using the wedge $W[a]$ again we can construct a group $U\left[a, \ell^{\prime}\right](s)$ in the same manner. By proper definition, this group satisfies again the spectrum condition and the relations similar to $(3,2)-(3.4)$. The only change is 


$$
\operatorname{ad} \Delta[a]^{\mathrm{i} t} U\left[a, \ell^{\prime}\right](s)=U\left[a, \ell^{\prime}\right](\mathrm{e}(-t) s) .
$$

Also here we obtain a group $U\left[\ell^{\prime}\right](s)$ which does not depend on the first parameter.

It remains to show that the groups $U[\ell](s)$ and $U\left[\ell^{\prime}\right](s)$ commute. To this end we notice that we can map $\mathscr{\mathscr { C }}(W[a])$ onto $\mathscr{M}\left(W\left[a+t \ell-s \ell^{\prime}\right]\right)$ on two different ways, namely using either $\mathscr{\mathscr { C }}(W[a+t \ell])$ or $\mathscr{\mathscr { C }}\left(W\left[a-s \ell^{\prime}\right]\right)$ as intermediate algebra. This yields

$$
\begin{aligned}
& \mathscr{M}\left(W\left[a+t \ell-s \ell^{\prime}\right]\right)=\operatorname{ad} U\left[\ell^{\prime}\right](-s) U[\ell](t) \mathscr{M}(W[a]), \\
& \mathscr{M}\left(W\left[a+t \ell-s \ell^{\prime}\right]\right)=\operatorname{ad} U[\ell](t) U\left[\ell^{\prime}\right](-s) \mathscr{M}(W[a]) .
\end{aligned}
$$

We want to show that the product of translation operators coincide. Therefore, we compute with help of (3.2) and (3.2') and obtain

$$
\begin{gathered}
U\left[a+t \ell, \ell^{\prime}\right](-s(1-\mathrm{e}(-\mu))) U[a, \ell](t(1-\mathrm{e}(\mu)))= \\
\Delta\left[a+t \ell-s \ell^{\prime}\right]^{\mathrm{i} \mu} \Delta[a+t \ell]^{-\mathrm{i} \mu} \Delta[a+t \ell]^{\mathrm{i} \mu} \Delta[a]^{-\mathrm{i} \mu} \\
U\left[a-s \ell^{\prime}, \ell\right](t(1-\mathrm{e}(\mu))) U\left[a, \ell^{\prime}\right](-s(1-\mathrm{e}(-\mu)))= \\
\Delta\left[a+t \ell-s \ell^{\prime}\right]^{\mathrm{i} \mu} \Delta\left[a-s \ell^{\prime}\right]^{-\mathrm{i} \mu} \Delta\left[a-s \ell^{\prime}\right]^{\mathrm{i} \mu} \Delta[a]^{-\mathrm{i} \mu} .
\end{gathered}
$$

Using the independence of the first parameter we obtain

$$
U\left[\ell^{\prime}\right](a) U[\ell](b)=U[\ell](b) U\left[\ell^{\prime}\right](a) .
$$

Having constructed the Poincaré group in two dimensions we have to go to higher dimensions. First we want to show that the translations defined in different twoplanes also commute. To this end we fix a lightlike direction $\ell$ and look at the family of wedges defined by $\ell$ and another lightlike vector $\left\{W\left[\ell, \ell^{\prime}\right] ; \ell^{\prime} \neq \ell\right\}$. Using the -half-sided modular inclusions $\mathscr{M}\left(W\left[\ell, \ell_{1}, \lambda \ell\right]\right) \subset \mathscr{M}\left(W\left[\ell, \ell_{1}, 0\right]\right)$ and $\mathscr{M}\left(W\left[\ell, \ell_{2}, \lambda \ell\right]\right) \subset \mathscr{M}\left(W\left[\ell, \ell_{2}, 0\right]\right)$ we obtain two different translation groups $U\left[\ell, \ell_{1}, \ell\right](t)$ and $U\left[\ell, \ell_{2}, \ell\right](t)$ respectively. Both groups act like translations on every double cone and hence on every wedge. Therefore, by Theorem 2.3 they have to coincide. Hence the groups depend only on the direction of the translations and not on the two-plane which has been used for constructing them. Consequently we obtain groups $U[\ell](t)$. From this it follows that all these groups $U[\ell](s)$ commute for different $\ell$, since for every two different $\ell$ 's there exists a wedge which is defined by these two vectors. Since all these unitary groups fulfill the spectrum condition there exists a group $V(a), a \in \mathbb{R}^{4}$ such that $U[\ell](s)$ coincides with $V(s \ell)$. Hence we have constructed the translation group of $\mathbb{R}^{4}$ which transforms by the modular groups in the expected way.

We collect the results obtained so far:

Lemma 3.1. Assume all modular groups of the wedge algebras act like their associated Lorentz groups. Then a unique continuous representation of the translation-group $V(a)$ exists which fulfills spectrum-condition and acts geometrically on the local algebras

$$
\operatorname{ad} V(a) \mathscr{M b}(K)=\mathscr{M}(K+a)
$$

where $K$ denotes a double cone. (It is assumed, that $\mathscr{M}(K)$ coincides with the intersection of the wedge algebras of all wedges containing $K$.) This representation $V(a)$ is contained in the algebra generated by the modular groups.

Moreover, the modular groups of the wedges and the translations transform each other as if they were members of a unitary representation of the Poincare group. 
Proof. We know that $V(a)$ transforms the algebras of the wedges in the geometric manner. This implies the correct action on $\mathscr{M}(K)$ by passing to the intersection. The rest follows from (3.2) and (3.2') and the fact that every translation can be decomposed into translations in lightlike directions.

From this result we obtain

Proposition 3.2. Let a representation of a theory of local observables fulfill the abovementioned conditions. Then this representation fulfills wedge duality, i.e.

$$
\mathscr{M}\left(W\left[\ell, \ell^{\prime}\right]\right)^{\prime}=\mathscr{M}\left(W\left[\ell^{\prime}, \ell\right]\right) .
$$

Proof. Since in every two-dimensional subspace associated with a wedge we have a representation of the Poincare group which acts local and since the Lorentz boosts coincide with the modular group it follows that for every localized operator $A$ belonging to the right wedge the expression $U(\Lambda(t)) A \Omega$ has a bounded analytic continuation into the strip $-\frac{1}{2}<\operatorname{Im} t<0$ with continuous boundary values. This follows from the fact that $A \Omega$ is in the domain of $\Delta^{\frac{1}{2}}$. Hence the conditions of [Bch3] are fulfilled and the theory obeys wedge duality.

Since an algebra and its commutant has up to a sign the same modular group we obtain the following symmetry:

$$
\Delta\left[\ell, \ell^{\prime}\right]^{\mathrm{i} t}=\Delta\left[\ell^{\prime}, \ell\right]^{-\mathrm{i} t} .
$$

\section{Construction of the Lorentz Group}

Our aim is to show that the operators $\Delta\left[\ell_{1}, \ell_{2}\right]^{\text {it }}$ generate a representation of the Lorentz group. Therefore, we have to show that

$$
\prod \Delta\left[\ell_{1}^{(i)}, \ell_{2}^{(\imath)}\right]^{\mathrm{i} t^{(\imath)}}=\mathbb{1}
$$

holds in case the equation

$$
\prod \Lambda\left[\ell_{1}^{(i)}, \ell_{2}^{(i)}\right]\left(t^{(i)}\right)=1
$$

is fulfilled. To show this we are only allowed to make transformations which do not change the conclusion, i.e. the transformations (1.6), (3.6) and those derived from this. We will find other transformations by looking at half-sided modular inclusions.

The first step of our construction will be to look at the family of wedges having one lightray in common,

$$
\left\{W\left[\ell, \ell_{2}\right] ; \ell \text { fixed }\right\},
$$

and the stabilizer group of the vector $\ell$. We have discussed this group in the section following formula (2.4)

Looking at Eq. (2.7') we see that we have to show that the corresponding product $\Delta\left[\ell, \ell_{2}(a)\right]^{\mathrm{it}} \Delta\left[\ell, \ell_{2}(b)\right]^{-\mathrm{i} t}$ converges for $t \rightarrow \infty$ strongly to a unitary operator $U^{\ell}(a, b)$ and that this operator acts local on every double cone, i.e.,

$$
\operatorname{ad} U^{\ell}(a, 0) \mathscr{M}(O)=\mathscr{M}\left(\Lambda^{\ell}(a) O\right) .
$$

Lemma 4.1. The product 


$$
\Delta\left[\ell, \ell_{2}(a)\right]^{\mathrm{i} t} \Delta\left[\ell, \ell_{2}(b)\right]^{-\mathrm{i} t}
$$

converges for $t \rightarrow \infty$ strongly to an operator $U^{\ell}(a, b)$. This operator acts geometrically on local algebras, i.e. with the notation of Sect. 2

$$
\operatorname{ad} U^{\ell}(a, b) \mathscr{M}(K)=\mathscr{M}\left(\Lambda^{\ell}(a-b) K\right) .
$$

Proof. Since by Lemma 2.6 $\mathscr{Q}\left(W\left[\ell, \ell_{2}(a)\right] \cap W\left[\ell, \ell_{2}(b)\right]\right)$ fulfills the condition of - half-sided modular inclusion with respect to the two algebras $\mathscr{M}\left(W\left[\ell, \ell_{2}(b)\right]\right)$ and $\mathscr{M}\left(W\left[\ell, \ell_{2}(a)\right]\right)$ there exist by Theorem 2.1 two one-parametric unitary groups $U^{\ell}[a, b ; a](t), U^{\ell}[a, b ; b](t)$ with the properties

$$
\mathscr{M}\left(W\left[\ell, \ell_{2}(a)\right] \cap W\left[\ell, \ell_{2}(b)\right]\right)=\left\{\begin{array}{l}
\operatorname{ad} U^{\ell}[a, b ; a](1) \mathscr{M}\left(W\left[\ell, \ell_{2}(a)\right]\right), \\
\operatorname{ad} U^{\ell}[a, b ; b](1) \mathscr{M}\left(W\left[\ell, \ell_{2}(b)\right]\right) .
\end{array}\right.
$$

Both these groups fulfill similar properties as listed in (3.2). From this we derive

$$
\operatorname{ad} U^{\ell}[a, b ; a](1)^{-1} U^{\ell}[a, b ; b](1) \mathscr{M}\left(W\left[\ell, \ell_{2}(b)\right]\right)=\mathscr{M}\left(W\left[\ell, \ell_{2}(a)\right]\right) .
$$

These operators are connected with the modular operators of the algebras and their intersections by the formulas

$$
\begin{aligned}
& U^{\ell}[a, b ; a](1-\mathrm{e}(t))=\Delta[\cap]^{\mathrm{i} t} \Delta\left[\ell, \ell_{2}(a)\right]^{-\mathrm{i} t}, \\
& U^{\ell}[a, b ; b](1-\mathrm{e}(t))=\Delta[\cap]^{\mathrm{i} t} \Delta\left[\ell, \ell_{2}(b)\right]^{-\mathrm{i} t},
\end{aligned}
$$

where $\Delta[\cap]$ denotes the modular operator of the intersection. We find

$$
U^{\ell}[a, b ; a](1-\mathrm{e}(t))^{-1} U^{\ell}[a, b ; b](1-\mathrm{e}(t))=\Delta\left[\ell, \ell_{2}(a)\right]^{\mathrm{i} t} \Delta\left[\ell, \ell_{2}(b)\right]^{-\mathrm{i} t} .
$$

This shows that for $t \rightarrow \infty$ the product on the left converges weakly and hence also strongly. Therefore, also the right-hand side converges strongly. Since the approximations $\Delta\left[\ell, \ell_{2}(a)\right]^{\mathrm{it}} \Delta\left[\ell, \ell_{2}(b)\right]^{-\mathrm{i} t}$ act geometrically we see with (3.6) that this is also true for the limit

$$
U^{\ell}(a, b)=\lim _{t \rightarrow \infty} U^{\ell}[a, b ; a](1-\mathrm{e}(t))^{-1} U^{\ell}[a, b ; b](1-\mathrm{e}(t)) .
$$

Equation (3.6) shows that the limit acts as stated in the lemma.

Next we have to show that the operators $U(a, b)$ depend only on the difference $(a-b)$ and that the operators $V(a-b)=U(a, b)$ define a representation of the two dimensional translation group.

Lemma 4.2. The operators $U^{\ell}(a, b)$ depend only on the difference of the arguments

$$
U^{\ell}(a, b)=V^{\ell}(a-b) .
$$

These operators define a continuous representation of the two-dimensional abelian group $\Lambda^{\ell}(a)$

$$
V^{\ell}(a) V^{\ell}(b)=V^{\ell}(a+b), \quad a, b \in \mathbb{R}^{2} .
$$

Proof. We first exploit Eq. (1.6) which leads to 


$$
\begin{aligned}
\operatorname{ad} \Delta\left[\ell, \ell_{2}(b)\right]^{\mathrm{i} t} \Delta\left[\ell, \ell_{2}(a)\right]^{\mathrm{i} s} & =\Delta\left[\ell, \ell_{2}(\mathrm{e}(t)(a-b)-b)\right]^{\mathrm{i} s}, \\
\operatorname{ad} \Delta\left[\ell, \ell_{2}(b)\right]^{\mathrm{i} t} U^{\ell}(a, c) & =U^{\ell}(\mathrm{e}(t)(a-b)+b, \mathrm{e}(t)(c-b)+b), \\
\operatorname{ad} U^{\ell}(a, c) \Delta\left[\ell, \ell_{2}(c)\right]^{\mathrm{i} s} & =\Delta\left[\ell, \ell_{2}(\mathrm{e}(t)(b-a)+c-b+a)\right]^{\mathrm{i} s}, \\
\operatorname{ad} U^{\ell}(a, b) U^{\ell}(c, d) & =U^{\ell}(c+a-b, d+a-b) .
\end{aligned}
$$

The first line follows from (1.6) together with (2.3') and (2.6). Replacing in the first line the second operator by a product and taking the limit we get the second line. The third line is obtained by iterating the first line and taking the limit again. The limiting procedure is well defined since $U^{\ell}(a, c)$ is the strong limit. So we obtain first the weak convergence but again by unitarity the strong convergence. The last line can easily be derived from the third line.

From the relation $U^{\ell}(a, b)=U^{\ell}[a, b ; a](1)^{-1} U^{\ell}[a, b ; b](1)$ we conclude

$$
U^{\ell}(a, b) U^{\ell}(b, a)=1 .
$$

Since the subalgebras

$$
\mathscr{M}\left(W\left[\ell, \ell_{2}(a)\right] \cap W\left[\ell, \ell_{2}(b)\right]\right) \quad \text { and } \mathscr{M}\left(W\left[\ell, \ell_{2}(a)\right] \cap W\left[\ell, \ell_{2}(c)\right]\right)
$$

fulfill both the condition of - half-sided modular inclusion with respect to the two algebras $\mathscr{M}\left(W\left[\ell, \ell_{2}(a)\right]\right)$ we see that the triple intersection $\mathscr{M}\left(W\left[\ell, \ell_{2}(a)\right] \cap\right.$ $\left.W\left[\ell, \ell_{2}(b)\right] \cap W\left[\ell, \ell_{2}(c)\right]\right)$ fulfills also the condition of - half-sided modular inclusion with respect to $\mathscr{M}\left(W\left[\ell, \ell_{2}(a)\right]\right)$. By symmetry the same holds with respect to the algebras $\mathscr{M}\left(W\left[\ell, \ell_{2}(b)\right]\right), \mathscr{M}\left(W\left[\ell, \ell_{2}(c)\right]\right)$. Hence we obtain as in the last lemma three groups $U^{\ell}[a, b, c ; a](t), U^{\ell}[a, b, c ; b](t), U^{\ell}[a, b, c ; c](t)$. Now we can represent $U^{\ell}(a, b)$ with help of these operators

$$
U^{\ell}(a, b)=U^{\ell}[a, b, c ; a](1)^{-1} U^{\ell}[a, b, c ; b](1) .
$$

This leads to the relation

$$
\begin{aligned}
& U^{\ell}(a, b) U^{\ell}(b, c) U^{\ell}(c, a)=1, \\
& U^{\ell}(a, b) U^{\ell}(b, c)=U^{\ell}(a, c) .
\end{aligned}
$$

For the second equation we have used (4.6). Now we start from the last equation of (4.6') and use the last line of (4.5),

$$
\begin{aligned}
& U^{\ell}(a, b) U^{\ell}(c, d)=U^{\ell}(a, b) U^{\ell}(c, b) U^{\ell}(b, d) \\
& \quad=U^{\ell}(c+a-b, a) U^{\ell}(a, b) U^{\ell}(b, d)=U^{\ell}(a+c-b, d) .
\end{aligned}
$$

Taking the inverse of this relation we obtain with (4.6)

$$
U^{\ell}(a, b) U^{\ell}(c, d)=U^{\ell}(a, d-(c-b)),
$$

whereby the arguments have been renamed. Comparing the last two equations, we get with $f=c-d$ the equation

$$
U^{\ell}(a+f, b)=U^{\ell}(a, b-f) .
$$

This shows that $U^{\ell}(a, b)$ depends only on the difference-variable. We set

$$
V^{\ell}(a-b)=U^{\ell}(a, b)=U^{\ell}(a-b, 0) .
$$


Inserting this into $(*)$ we find

$$
V^{\ell}(a-b) V^{\ell}(c-d)=V^{\ell}(a-d+c-b) .
$$

Hence the $V^{\ell}(a)$ define an abelian representation of the two-dimensional translation group.

It remains to show that this is a continuous representation. The second line of (4.5) together with (4.7) gives

$$
\operatorname{ad} \Delta\left[\ell, \ell_{2}(b)\right]^{\mathrm{i} t} V^{\ell}(a)=V^{\ell}(\mathrm{e}(t) a) .
$$

Since the modular group is continuous we see that $V^{\ell}(a)$ is continuous in radial direction. Multiplying this expression with $V(b)$ we see that $V(\mathrm{e}(t) a+b)$ is continuous in $t$ for every value of $a$ and $b$. Hence $V(a)$ is continuous.

With the operators $V^{\ell}(a)$ and $\Delta\left[\ell, \ell_{2}(b)\right]^{\mathrm{it}}$ we can construct the group $G(\ell)$ generated by these elements. These groups have three generators and will be described in Corollary 4.3. While the groups $V^{\ell}(a)$ have a trivial intersection for different $\ell$ this is no longer true for the groups $G(\ell)$ and $G\left(\ell^{\prime}\right)$. The intersection consists of the elements $\Delta\left[\ell, \ell^{\prime}\right]^{i t}$. Since the modular groups of the corresponding wedges are unique there is no obstruction between the representations of the different $G(\ell)$. The multiplication-rule of the group $G(\ell)$ is the following

$$
\Delta\left[\ell, \ell_{2}(0)\right]^{\mathrm{i} t} V^{\ell}(a) \Delta\left[\ell, \ell_{2}(0)\right]^{\mathrm{i} s} V^{\ell}(b)=\Delta\left[\ell, \ell_{2}(0)\right]^{\mathrm{i}(t+s)} V^{\ell}(\mathrm{e}(-s) a+b) .
$$

Corollary 4.3. The elements of the form

$$
\Delta\left[\ell, \ell_{2}(0)\right]^{\mathrm{it}} V^{\ell}(a)
$$

yield a continuous representation of the group generated by $\left\{\Lambda^{\ell}(a), \Lambda\left[\ell, \ell_{2}(b)\right\}\right.$ with the multiplication rule (4.9).

Proof. We know that $V^{\ell}(a)$ maps $\mathscr{M}\left(W\left[\ell, \ell_{2}(0)\right]\right)$ onto $\mathscr{M}\left(W\left[\ell, \ell_{2}(a)\right]\right)$. From this follows together with (4.8)

$$
\Delta\left[\ell, \ell_{2}(a)\right]^{\mathrm{i} s}=V^{\ell}(a) \Delta\left[\ell, \ell_{2}(0)\right]^{\mathrm{i} s} V^{\ell}(-a)=\Delta\left[\ell, \ell_{2}(0)\right]^{\mathrm{i} s} V^{\ell}(\mathrm{e}(t) a-a) .
$$

Hence by (4.8) we get a group with the stated product rule.

Using the groups $G(\ell)$ and $G\left(\ell^{\prime}\right)$ we will make a transformation of (4.1) in such a way that there appears only a product of two types of elements such that the factors belong either to $G(\ell)$ or to $G\left(\ell^{\prime}\right)$. We will call two products equivalent if they are transformed with help of (1.6) and (3.6) or with elements derived from this. First we show

Lemma 4.4. Let $\ell, t, \ell^{\prime}$ lie in a two-plane. Then every element $\Lambda\left[\ell_{1}, \ell_{2}\right](t)$ is equivalent to a product of the form

$$
\operatorname{ad}\left\{\Lambda^{\ell}(a) \Lambda^{\ell^{\prime}}(b)\right\} \Lambda\left[\ell, \ell^{\prime}\right]\left(t^{\prime}\right)
$$

where $t^{\prime}$ is either $t$ or $-t$.

Proof. We look at the transformation $\Lambda\left[\ell_{1}, \ell_{2}\right](t)$. If $\ell_{1}=\ell$ and $\ell_{2}=\ell^{\prime}$ then we get the lemma with $a=b=0$. If $\ell_{1}=\ell^{\prime}$ and $\ell_{2}=\ell$ then we use (3.6) for transforming the element to the previous situation. If one of the two vectors $\ell_{1}, \ell_{2}$ coincides with $\ell$ we can assume that this is $\ell_{1}$. Then there is a transformation $\Lambda^{\ell}(a)$ mapping $\ell_{2}$ onto 
a multiple of $\ell^{\prime}$. Therefore, $\Lambda\left[\ell, \ell_{2}\right](t)$ is equivalent to ad $\Lambda^{\ell}(a) \Lambda\left[\ell, \ell^{\prime}\right](t)$. If one of the two vectors $\ell_{1}, \ell_{2}$ coincides with $\ell^{\prime}$ we can assume that this is $\ell_{2}$. By the same argument we find that $\Lambda\left[\ell, \ell_{2}\right](t)$ is equivalent to ad $\Lambda^{\ell^{\prime}}(b) \Lambda\left[\ell, \ell^{\prime}\right](t)$.

Assume next that $\ell_{1}$ and $\ell_{2}$ are not multiples of $\ell$ or $\ell^{\prime}$. Then there is a transformation $\Lambda^{\ell}(a)$ mapping $\ell_{2}$ onto a multiple of $\ell^{\prime}$. By this transformation $\ell_{1}$ is mapped onto $\ell_{3}$. Hence we get a transformation $\Lambda^{\ell^{\prime}}(b)$ which maps $\ell_{3}$ onto a multiple of $\ell$. Since this transformation does not change $\ell^{\prime}$ the original transformation is mapped onto $A\left[\ell, \ell^{\prime}\right](t)$. If one of the vectors is already in the right position then we need only one transformation. If necessary we can change the order of vectors because of (3.6). Hence every element $\Lambda\left[\ell_{1}, \ell_{2}\right](t)$ is equivalent to an element of the form

$$
\operatorname{ad}\left\{\Lambda^{\ell}(a) \Lambda^{\ell^{\prime}}(b)\right\} \Lambda\left[\ell, \ell^{\prime}\right]\left(t^{\prime}\right)
$$

where $t^{\prime}$ is either $t$ or $-t$.

Using this lemma we show:

Lemma 4.5. Let $\ell, t, \ell^{\prime}$ lie in a two-plane then every product

$$
\prod_{i=1}^{n} \Lambda\left[\ell_{1}^{(i)}, \ell_{2}^{(i)}\right]\left(t^{(i)}\right)
$$

is equivalent to the product

$$
\Lambda\left[\ell, \ell^{\prime}\right]\left(t^{(0)}\right) \prod_{i=1}^{m} \Lambda^{\ell^{\prime}}\left(b^{(i)}\right) \Lambda^{\ell}\left(a^{(i)}\right) .
$$

Proof. Using the last lemma we replace every $\Lambda\left[\ell_{1}^{(i)}, \ell_{2}^{(i)}\right]\left(t^{(i)}\right)$ by an element of the form ad $\left\{\Lambda^{\ell}\left(a^{\prime}(i)\right) \Lambda^{\ell^{\prime}}\left(b^{\prime}(i)\right)\right\} \Lambda\left[\ell, \ell^{\prime}\right]\left(t^{\prime}(i)\right)$. Using (4.8) in the form

$$
\begin{aligned}
\operatorname{ad} \Lambda\left[\ell, \ell^{\prime}\right](t) \Lambda^{\ell}(a) & =\Lambda^{\ell}(\mathrm{e}(t) a), \\
\operatorname{ad} \Lambda\left[\ell, \ell^{\prime}\right](t) \Lambda^{\ell^{\prime}}(a) & =\Lambda^{\ell^{\prime}}(\mathrm{e}(-t) a)
\end{aligned}
$$

we can commute all $\Lambda\left[\ell, \ell^{\prime}\right]\left(t^{\prime}(i)\right)$ to the front and multiply them. Therefore, we end up with an expression listed in the lemma. Since the $\Lambda^{\ell}(a)$ and the $\Lambda^{\ell^{\prime}}(a)$ are groups all arguments are unequal to zero except perhaps for the first $\Lambda^{\ell^{\prime}}(b)$ or the last $\Lambda^{\ell}(a)$.

Using this lemma we have to investigate expressions of the form (4.11). For further simplification of this expression we must investigate the rotations.

Let $\Lambda^{\ell}(a)$ be an element in the stabilizer group of $\ell$. Then $\Lambda^{\ell}(a) t$ is a vector to which we can apply $\Lambda^{\ell^{\prime}}(b)$. There will be an element $b(a)$ such that $\Lambda^{\ell^{\prime}}(b(a)) \Lambda^{\ell}(a) t$ belongs to the two-plane containing $\ell, \ell^{\prime}$ and $t$. In this situation $s(a)$ exists such that $\Lambda\left[\ell, \ell^{\prime}\right](s(a))$ maps this vector back to $t$. Therefore, the product represents a rotation

$$
\Lambda\left[\ell, \ell^{\prime}\right](s(a)) \Lambda^{\ell^{\prime}}(b(a)) \Lambda^{\ell}(a)=R^{1}(a) .
$$

In the same manner we obtain a second rotation if we start with $\Lambda^{\ell^{\prime}}(a)$,

$$
\Lambda\left[\ell, \ell^{\prime}\right]\left(s^{\prime}(a)\right) \Lambda^{\ell}\left(b^{\prime}(a)\right) \Lambda^{\ell^{\prime}}(a)=R^{2}(a) .
$$


First we need to determine $b(a), s(a)$ and the element $R^{1}(a)$ and $b^{\prime}(a), s^{\prime}(a)$ and $R^{2}(a)$ respectively. This we do in our standard coordinate system. $\Lambda^{\ell}(a)$ maps the vector $t$ onto $\Lambda^{\ell}(a) t=\left(1+\frac{a^{2}}{2}, \frac{a^{2}}{2}, a_{1}, a_{2}\right)$ and hence we get

$$
\begin{aligned}
\Lambda^{\ell^{\prime}}(b) & \Lambda^{\ell}(a) t=\left(\left(1+\frac{b^{2}}{2}\right)\left(1+\frac{a^{2}}{2}\right)+\frac{b^{2}}{2} \frac{a^{2}}{2}+(b, a),\right. \\
& \left.-\frac{b^{2}}{2}\left(1+\frac{a^{2}}{2}\right)+\left(1-\frac{b^{2}}{2}\right) \frac{a^{2}}{2}-(b, a), b_{1}\left(1+a^{2}\right)+a_{1}, b_{2}\left(1+a^{2}\right)+a_{2}\right) .
\end{aligned}
$$

This vector belongs to the plane spanned by $\ell$ and $\ell^{\prime}$ for

$$
b(a)=-\frac{a}{1+a^{2}} .
$$

Inserting this we find

$$
\Lambda^{\ell^{\prime}}(b(a)) \Lambda^{\ell}(a) t=\left(\frac{2+2 a^{2}+a^{4}}{2\left(1+a^{2}\right)}, \frac{2 a^{2}+a^{4}}{2\left(1+a^{2}\right)}, 0,0\right) .
$$

This implies

$$
\Lambda\left[\ell, \ell^{\prime}\right](s(a))=\frac{1}{2\left(1+a^{2}\right)}\left(\begin{array}{cccc}
2+2 a^{2}+a^{4} & -\left(2 a^{2}+a^{4}\right) & 0 & 0 \\
-\left(2 a^{2}+a^{4}\right) & 2+2 a^{2}+a^{4} & 0 & 0 \\
0 & 0 & 1 & 0 \\
0 & 0 & 0 & 1
\end{array}\right)
$$

from which follows

$$
\mathrm{e}(s(a))=\frac{1}{\left(1+a^{2}\right)}
$$

In order to compute the rotation $R^{1}(a)$ notice first that $\Lambda^{\ell}(a)$ leaves the vector $(1,0,0) \times\left(0, a_{1}, a_{2}\right)$ unchanged. The same holds for $\Lambda^{\ell^{\prime}}\left(-\frac{a}{1+a^{2}}\right)$ and $\Lambda\left[\ell, \ell^{\prime}\right](s(a))$ so that $\frac{1}{\|a\|}(1,0,0) \times\left(0, a_{1}, a_{2}\right)$ is the axis of rotation. (The multiplication is the vectorproduct in $\mathbb{R}^{3}$.) The angle of rotation can be computed by applying $R^{1}(a)$ to the vector $(0,1,0,0)$. One finds

$$
\begin{aligned}
& \Lambda\left[\ell, \ell^{\prime}\right](s(a)) \Lambda^{\ell^{\prime}}(b(a)) \Lambda^{\ell}(a)(0,1,0,0)= \\
& \quad \frac{1}{1+a^{2}}\left(0,1-a^{2},-2 a_{1},-2 a_{2}\right)=\frac{1-a^{2}}{1+a^{2}}(0,1,0,0)-\frac{2 a^{2}}{1+a^{2}}\left(0,0, \frac{a_{1}}{\|a\|}, \frac{a_{2}}{\|a\|}\right) .
\end{aligned}
$$

This implies the following characterization of $R^{1}(a)$

$$
R^{1}(a):\left\{\begin{array}{l}
\text { axis of rotation : } \quad \frac{1}{\|a\|}(1,0,0) \times\left(0, a_{1}, a_{2}\right), \\
\text { angle of rotation }: \cos \varphi=\frac{1-a^{2}}{1+a^{2}}, \sin \varphi=-\frac{2\|a\|}{1+a^{2}} .
\end{array}\right.
$$

By similar computation one finds 


$$
\begin{aligned}
b^{\prime}(a) & =\frac{a}{1+a^{2}}, \\
\Lambda\left[\ell, \ell^{\prime}\right]\left(s^{\prime}(a)\right) & =\frac{1}{2\left(1+a^{2}\right)}\left(\begin{array}{cccc}
2+2 a^{2}+a^{4} & \left(2 a^{2}+a^{4}\right) & 0 & 0 \\
\left(2 a^{2}+a^{4}\right) & 2+2 a^{2}+a^{4} & 0 & 0 \\
0 & 0 & 1 & 0 \\
0 & 0 & 0 & 1
\end{array}\right), \\
\mathrm{e}\left(s^{\prime}(a)\right. & =\left(1+a^{2}\right), \\
R^{2}(a) & : \begin{cases}\text { axis of rotation : } & \frac{1}{\|a\|}(1,0,0) \times\left(0, a_{1}, a_{2}\right), \\
\text { angle of rotation : } & \cos \varphi=\frac{1-a^{2}}{1+a^{2}}, \sin \varphi=\frac{2\|a\|}{1+a^{2}} .\end{cases}
\end{aligned}
$$

Fixing the axis of rotation and replacing in (4.12) and (4.12') the Lorentz transformations by its representants then we obtain a family of representations

$$
U(R(\ell, d, \varphi))=\Delta\left[\ell, \ell^{\prime}\right]^{i s(a)} V^{\ell^{\prime}}\left(-\frac{a}{1+a^{2}}\right) V^{\ell}(a) .
$$

In this formula $d$ means the normalized rotation axis. The angle $\varphi$ does not admit the value $\pi$. In the original definition $\varphi$ was non-negative, but we can drop this restriction by identifying $R(\ell, d,-\varphi)$ with $R(\ell,-d, \varphi)$.

Next we investigate the rotations defined in (4.15). First we show:

Lemma 4.6. The operators $U(R(\ell, d, \varphi))$ defined in Eq. (4.15) do not depend on the argument $\ell$. They are continuous in the direction $d$ and in the angle provided $-\pi<\varphi<\pi$.

Proof. First we show that $U(R(\ell, d, \psi))$ is continuous in $\psi$. Notice first that $V^{\ell^{\prime}}\left(-\frac{a}{1+a^{2}}\right) V^{\ell}(a)$ is weakly continuous in $a$ and by the unitarity of the product also strongly continuous. Repeating this argument we find that the expression $\Delta\left[\ell, \ell^{\prime}\right]^{i s(a)} V^{\ell^{\prime}}\left(-\frac{a}{1+a^{2}}\right) V^{\ell}(a)$ is continuous in $a$. If we keep the direction of $a$ fixed then we obtain that $U(R(\ell, d, \psi))$ is continuous in $\psi$.

Next we show that the expression $U(R(\ell, d, \varphi))$ depends continuously on $\ell$. Notice first that (1.6) and the definition of $U(R(\ell, d, \psi))$ implies the relation

$$
\operatorname{ad} U(R(\ell, d, \psi)) \Delta\left[\ell_{1}, \ell_{2}\right]^{\mathrm{i} t}=\Delta\left[R(\ell, d, \psi) \ell_{1}, R(\ell, d, \psi) \ell_{2}\right]^{\mathrm{i} t} .
$$

Consequently (4.15) implies

$$
\operatorname{ad} U(R(\ell, d, \psi)) U\left(R\left(\ell_{1}, d, \varphi\right)=U\left(R\left(R(\ell, d, \psi) \ell_{1}, d, \varphi\right)\right)\right.
$$

in case $\ell_{1}$ is perpendicular to $d$. From this we obtain continuity in $\ell$ since we know the continuity of $U(R(\ell, d, \psi))$ in $\psi$.

Let now $\varphi$ be an irrational multiple of $2 \pi$. Then $\{n \varphi \bmod 2 \pi ; n \in \mathbb{Z}\}$ is dense in the open interval $(-\pi, \pi)$. Choosing $\psi=\varphi$ in (4.15') and $\ell_{1}=\ell$ then we obtain

$$
U(R(\ell, d, \varphi))=U(R(R(\ell, d, \varphi) \ell, d, \varphi))
$$

Iterating this equation we get:

$$
\begin{aligned}
U(R(\ell, d, \varphi)) & =U\left(R\left(R^{n}(\ell, d, \varphi) \ell, d, \varphi\right)\right) \\
& =U(R(R(\ell, d, n \varphi) \ell, d, \varphi)), \quad n \in \mathbb{Z} .
\end{aligned}
$$


Using the continuity in $\ell$ we find that $U(R(\ell, d, \varphi))$ is independent of $\ell$, provided $\varphi / 2 \pi$ is irrational. Since $U(R(\ell, d, \varphi))$ is continuous in $\varphi$ it follows the independence of $\ell$ for all $\varphi$. Since $U(R(d, \varphi))$ is continuous in $\varphi$ we conclude from

$$
\operatorname{ad} U\left(R\left(d^{\prime}, \varphi\right)\right) U(R(d, \psi))=U\left(R\left(R\left(d^{\prime}, \varphi\right) d, \psi\right)\right)
$$

that $U(R(d, \varphi))$ is also continuous at $d$ in any direction. Since this is true for any point $d$ on the unit-sphere we obtain continuity in $d$.

Knowing the identity of the different representations of the rotations we can make a further transformation of the expression (4.11).

Lemma 4.7. The expression (4.11) is equivalent to one of the expressions

$$
\begin{aligned}
& \Lambda\left[\ell, \ell^{\prime}\right]\left(t_{0}\right) \Lambda^{\ell}\left(a_{0}\right) \prod_{i=1}^{m} R(\ell, d(i), \varphi(i)), \\
& \Lambda\left[\ell, \ell^{\prime}\right]\left(t_{0}\right) \Lambda^{\ell^{\prime}}\left(a_{0}\right) \prod_{i=1}^{m} R(\ell, d(i), \varphi(i)) .
\end{aligned}
$$

Proof. Assume that at the end of (4.11) there is an element $\Lambda^{\ell}(a)$. We can replace it by $\Lambda^{\ell^{\prime}}\left(\frac{a}{1+a^{2}}\right) \Lambda\left[\ell, \ell^{\prime}\right](-s(a)) \Lambda\left[\ell, \ell^{\prime}\right](s(a)) \Lambda^{\ell^{\prime}}\left(-\frac{a}{1+a^{2}}\right) \Lambda^{\ell}(a)$. The last three factors give rise to an element $R(\ell, d(a), \varphi(a))$. By using (4.8') the $\Lambda$ factor can be commuted to the left. The remaining $\Lambda^{\ell^{\prime}}$ factor can be combined with the factor of the same kind which was to the left of $\Lambda^{\ell}(a)$. Therefore, at the end we find after these manipulations an expression of the form $\Lambda^{\ell^{\prime}}(b) R(\ell, d(a), \varphi(a))$. Now we can perform with $\Lambda^{\ell^{\prime}}(b)$ the similar manipulation and obtain a factor $R\left(\ell^{\prime}, d(b), \varphi(b)\right)$. This can be replaced by $R(\ell, d(b),-\varphi(b))$. So we obtained for the last two factors of (4.11) the factors $R(\ell, d(b),-\varphi(b)) R(\ell, d(a), \varphi(a))$. Repeating this procedure we end up with one of the expressions (4.16). If there is an element $\Lambda^{\ell^{\prime}}(b)$ at the end of (4.11) the procedure is the same.

We are interested in the situation where the expression (4.16) is of the value 1. In this situation (4.16) can be simplified.

Lemma 4.8. Assume (4.16) has the value 1 . Then one finds $\Lambda\left[\ell, \ell^{\prime}\right]\left(\mathrm{i} t_{0}\right)=1$ and $\Lambda^{\ell}\left(a_{0}\right)=1$ and $\Lambda^{\ell^{\prime}}\left(a_{0}\right)=1$.

Proof. We consider the first line of (4.16). Since the product has the value 1 it follows that $\ell$ is mapped onto itself. Since the first two factors leave the direction of $\ell$ unchanged the same must be true for the product of the rotations. But this implies that the product of the rotations, which does not change $t$, maps $\ell$ onto itself. Hence we get $\Lambda\left[\ell, \ell^{\prime}\right]\left(t_{0}\right) \ell=\ell$ which implies $t_{0}=0$. Since the product of the rotations maps $\ell$ onto itself it also keeps $\ell^{\prime}$ fixed, which must be true also for $\Lambda^{\ell}\left(a_{0}\right)=1$. This implies $a_{0}=0$. The second line of (4.16) can be handled in the same manner.

Knowing that $U(R(d, \varphi))$ depends only on the direction of the axis of rotation and the rotation angle we have to show that these operators form for fixed axis of rotation a representation of the circle group.

Proposition 4.9. For fixed axis of rotation the operators $U(R(d, \varphi))$ give rise to a representation of the rotation group. This implies in particular that 


$$
U(R(d, \pi))=\lim _{\varphi \nearrow \pi} U(R(d, \varphi))
$$

exists and $U(R(d, \varphi))$ is continuous in $\varphi$ on the whole circle.

Since the proof of this proposition is straightforward but lengthy we will present it in the appendix.

Now we are prepared for the main result.

Theorem 4.10. Assume the modular group of every wedge algebra $\mathscr{M}\left(W\left[\ell_{1}, \ell_{2}, a\right]\right)$ acts on every algebra of a double cone like the associated group of Lorentz boosts. Then the modular groups $\Delta^{\mathrm{i} t}\left[\ell_{1}, \ell_{2}, a\right]$ define a representation of the Poincaré group.

Proof. In Sect. 3 we have constructed the translation so that it remains to construct the Lorentz transformations. To this end we have to show that the equation $\prod \Lambda\left[\ell_{1}^{(i)}, \ell_{2}^{(i)}\right]\left(t^{(i)}\right)=1$ implies the relation $\prod \Delta\left[\ell_{1}^{(i)}, \ell_{2}^{(i)}\right]^{i t^{(i)}}=\mathbb{1}$. We saw in (4.11) that the product can be transformed into

$$
\Lambda\left[\ell, \ell^{\prime}\right]\left(t^{(0)}\right) \prod_{i=1}^{m} \Lambda^{\ell^{\prime}}\left(b^{(i)}\right) \Lambda^{\ell}\left(a^{(i)}\right)=1 .
$$

To show this the principle of half-sided modular inclusion was needed. Using Lemma 4.8 and Lemma 4.9 the product in question can be transformed into $\prod R(\ell, d(i), \varphi(i))=$ 1. So it remains to show that this implies $\prod U(R(\ell, d(i), \varphi(i))=\mathbb{1}$. From the relation

$$
\operatorname{ad} U\left(R\left(d_{1}, \varphi_{1}\right)\right) U\left(R\left(d_{2}, \varphi_{2}\right)=U\left(R\left(R\left(d_{1}, \varphi_{1}\right) d_{2}, \varphi_{2}\right)\right.\right.
$$

it follows that the operators $U(R(d, \varphi))$ give rise at most to a central extension of the rotation group. Since we know that the representations are unique for the rotations around a fixed axis we conclude by Mackey's method of induced representations [Mac] that the $U(R(d, \varphi))$ form a single valued representation of the whole rotation group. Hence follows $\prod U(R(\ell, d(i), \varphi(i))=\mathbb{1}$.

\section{Appendix}

Proof of Proposition 4.9. Due to the independence of $U(R(\ell, d, \varphi))$ from $\ell$ we obtain with $a^{2}=1$ the relation

$$
\Delta\left[\ell, \ell^{\prime}\right]^{\mathrm{i} s(\alpha a)} V^{\ell^{\prime}}\left(-\frac{\alpha a}{1+\alpha^{2}}\right) V^{\ell}(\alpha a)=\Delta\left[\ell, \ell^{\prime}\right]^{-\mathrm{i} s(\alpha a)} V^{\ell}\left(\frac{\alpha a}{1+\alpha^{2}}\right) V^{\ell^{\prime}}(-\alpha a) .
$$

Applying ad $\Delta\left[\ell, \ell^{\prime}\right]^{i t}$ to this relation we find by (4.8')

$$
\Delta\left[\ell, \ell^{\prime}\right]^{2 \mathrm{i} s(s)} V^{\ell^{\prime}}\left(-\frac{\mathrm{e}(-t) \alpha a}{1+\alpha^{2}}\right) V^{\ell}(\mathrm{e}(t) \alpha a)=V^{\ell}\left(\frac{\mathrm{e}(t) \alpha a}{1+\alpha^{2}}\right) V^{\ell^{\prime}}(-\mathrm{e}(-t) \alpha a) .
$$

Notice: If we fix the vector $\ell$ and the axis of rotation $d$ then we have also fixed $a$. Therefore, we obtain

$$
\Delta\left[\ell, \ell^{\prime}\right]^{\mathrm{i} s(\lambda a)} V^{\ell^{\prime}}\left(-\frac{\lambda a}{1+\lambda^{2}}\right) V^{\ell}(\lambda a) \Delta\left[\ell, \ell^{\prime}\right]^{\mathrm{is}(\mu a)} V^{\ell^{\prime}}\left(-\frac{\mu a}{1+\mu^{2}}\right) V^{\ell}(\mu a)
$$

for the product of two rotations around the same axis. Using (4.8') this expression becomes 


$$
\Delta\left[\ell, \ell^{\prime}\right]^{\mathrm{i}(s(\lambda a)+s(\mu a))} V^{\ell^{\prime}}\left(-\frac{\mathrm{e}(s(\mu a)) \lambda a}{1+\lambda^{2}}\right) V^{\ell}(\mathrm{e}(-s(\mu a)) \lambda a) V^{\ell^{\prime}}\left(-\frac{\mu a}{1+\mu^{2}}\right) V^{\ell}(\mu a) .
$$

We want to apply formula (4.17') to the third and fourth factor of the expression (4.18'). This implies the following identifications:

$$
\begin{aligned}
\mathrm{e}(-t) \alpha & =\frac{\mu}{1+\mu^{2}} \\
\frac{\mathrm{e}(t) \alpha}{1+\alpha^{2}} & =\mathrm{e}(-s(\mu a)) \lambda=\lambda\left(1+\mu^{2}\right) .
\end{aligned}
$$

For the last transformation we have used (4.13"). Since the left-hand sides have the same sign, this must also hold for the right-hand sides. Hence we get the restriction $\lambda \mu>0$. We can solve (4.19) and obtain

$$
\begin{aligned}
1+\alpha^{2} & =\frac{1}{1-\lambda \mu}, \\
\mathrm{e}(t) \alpha & =\frac{\lambda\left(1+\mu^{2}\right)}{1-\lambda \mu}, \quad \lambda \mu \neq 1, \lambda \mu>0 \\
\frac{\mathrm{e}(-t) \alpha}{1+\alpha^{2}} & =\frac{\mu(1-\lambda \mu)}{1+\mu^{2}} .
\end{aligned}
$$

Inserting (4.19') into (4.18') then the expression (4.18) obtains the form

$$
\begin{gathered}
\Delta\left[\ell, \ell^{\prime}\right]^{\mathrm{i}(s(\lambda a)+s(\mu a))} V^{\ell^{\prime}}\left(-\frac{\lambda a}{\left(1+\lambda^{2}\right)\left(1+\mu^{2}\right)}\right) \Delta\left[\ell, \ell^{\prime}\right]^{2 \mathrm{i}(s(\alpha a)} \\
\times V^{\ell^{\prime}}\left(-\frac{\mu(1-\lambda \mu)}{1+\mu^{2}} a\right) V^{\ell}\left(\frac{\lambda\left(1+\mu^{2}\right)}{1-\lambda \mu} a\right) V^{\ell}(\mu a) \\
\left.\left.=\Delta\left[\ell, \ell^{\prime}\right]^{2 \mathrm{i}(s(\alpha a)+2 s(\alpha a)} V^{\ell^{\prime}}\left(-\left[\frac{\mathrm{e}(2 s(\alpha a)) \lambda a}{\left(1+\lambda^{2}\right)\left(1+\mu^{2}\right)}\right)+\frac{\mu(1-\lambda \mu)}{1+\mu^{2}} a\right)\right] a\right) \\
\times V^{\ell}\left(\left[\frac{\lambda\left(1+\mu^{2}\right)}{1-\lambda \mu}+\mu\right] a\right) .
\end{gathered}
$$

The argument of the operator $V^{\ell}$ becomes $\frac{\lambda+\mu}{1-\lambda \mu}$. For computing the argument of $V^{\ell^{\prime}}$ notice first the relation

$$
\mathrm{e}(2 s(\alpha a))=\mathrm{e}(s(\alpha a))^{2}=\frac{1}{\left(1+\alpha^{2}\right)^{2}}=(1-\lambda \mu)^{2} .
$$

Inserting this we find

$$
\begin{aligned}
\left.\mathrm{e}(2 s(\alpha a)) \frac{\lambda}{\left(1+\lambda^{2}\right)\left(1+\mu^{2}\right)}+\frac{\mu(1-\lambda \mu)}{1+\mu^{2}}\right) & =\frac{\lambda \mu}{1-\lambda \mu} \frac{(1-\lambda \mu)^{2}}{\left(1+\lambda^{2}\right)\left(1+\mu^{2}\right)} \\
& =\frac{\frac{\lambda+\mu}{1-\lambda \mu}}{1+\left(\frac{\lambda+\mu}{1-\lambda \mu}\right)^{2}} .
\end{aligned}
$$

If we set

$$
\frac{\lambda+\mu}{1-\lambda \mu}=\rho
$$


then the product (4.18) becomes

$$
\Delta\left[\ell, \ell^{\prime}\right]^{\mathrm{i}(s(\lambda a)+s(\mu a)+2 s(\alpha a))} V^{\ell^{\prime}}\left(-\frac{\rho}{1+\rho^{2}}\right) V^{\ell}(\rho) .
$$

Finally it remains to look at the exponent of the modular operator. We know e $(s(\lambda a))=$ $\frac{1}{1+\lambda^{2}}$, which implies $s(\lambda a)=-\log \left(1+\lambda^{2}\right)$. Hence we obtain

$$
\begin{aligned}
& s(\lambda a)+s(\mu a)+2 s(\alpha a)=-\log \left(1+\lambda^{2}\right)\left(1+\mu^{2}\right)\left(1+\alpha^{2}\right)^{2} \\
& \quad=-\log \frac{\left(1+\lambda^{2}\right)\left(1+\mu^{2}\right)}{(1-\lambda \mu)^{2}}=-\log \left(1+\left(\frac{\lambda+\mu}{1-\lambda \mu}\right)^{2}\right)=-\log \left(1+\rho^{2}\right) .
\end{aligned}
$$

Since $\rho$ is symmetric in $\lambda$ and $\mu$ it follows that the rotations around a fixed axis commute and give rise to a rotation (provided $\lambda \mu \neq 1$ ). It remains to show that the relation $\varphi(\lambda a)+\varphi(\mu a)=\varphi(\rho a)$ is fulfilled. From $\left(4.13^{\prime \prime \prime}\right)$ we obtain $\mathrm{e}^{\mathrm{i} \varphi(\lambda a)}=\frac{(1-\mathrm{i} \lambda)^{2}}{1+\lambda^{2}}$ from which we get

$$
\mathrm{e}^{\mathrm{i} \varphi(\lambda a)} \mathrm{e}^{\mathrm{i} \varphi(\mu a)}=\frac{(1-\mathrm{i} \lambda)^{2}}{1+\lambda^{2}} \frac{(1-\mathrm{i} \mu)^{2}}{1+\mu^{2}}=\frac{\left(1-\mathrm{i} \frac{\lambda+\mu}{1-\lambda \mu}\right)^{2}}{1+\left(\frac{\lambda+\mu}{1-\lambda \mu}\right)^{2}} .
$$

This shows that the group-relations are fulfilled.

The restriction for the calculation was $\lambda \mu>0$ and $\lambda \mu \neq 1$. Therefore, we have to look at the angle $\pi$ and at the product with different signs of the angle. Let us regard the second problem first. We find with (4.13") and (4.17)

$$
\begin{gathered}
\left\{\Delta\left[\ell, \ell^{\prime}\right]^{\mathrm{i} s(\alpha a)} V^{\ell^{\prime}}\left(-\frac{\alpha a}{1+\alpha^{2}}\right) V^{\ell}(\alpha a)\right\}^{-1}=V^{\ell}(-\alpha a) V^{\ell^{\prime}}\left(-\frac{\alpha a}{1+\alpha^{2}}\right) \Delta\left[\ell, \ell^{\prime}\right]^{-\mathrm{i} s(\alpha a)} \\
=\Delta\left[\ell, \ell^{\prime}\right]^{-\mathrm{i} s(\alpha a)} V^{\ell}(-\mathrm{e}(s(\alpha a)) \alpha a) V^{\ell^{\prime}}\left(\mathrm{e}(-s(\alpha a)) \frac{\alpha a}{1+\alpha^{2}}\right) \\
=\Delta\left[\ell, \ell^{\prime}\right]^{-\mathrm{i} s(\alpha a)} V^{\ell}\left(-\frac{\alpha a}{1+\alpha^{2}}\right) V^{\ell^{\prime}}(\alpha a) \\
=\Delta\left[\ell, \ell^{\prime}\right]^{-\mathrm{i} s(\alpha a)} \Delta\left[\ell, \ell^{\prime}\right]^{2 \mathrm{i} s(\alpha a)} V^{\ell^{\prime}}\left(\frac{\alpha a}{1+\alpha^{2}}\right) V^{\ell}(-\alpha a) .
\end{gathered}
$$

This implies

$$
R(d, \varphi)^{-1}=R(d,-\varphi), \quad-\pi<\varphi<\pi
$$

From this we obtain the multiplication rule $(\varphi>\psi)$ :

$$
R(d, \varphi) R(d,-\psi)=R(d, \varphi-\psi) R(d, \psi) R(d,-\psi)=R(d, \varphi-\psi) .
$$

A similar calculation is valid for $\varphi<\psi$. We have to discuss the point $\varphi=\pi$.

We define $R(d, \pi):=R(d, \pi / 2)^{2}$. Since $R(d, \varphi)$ is continuous in $\varphi$ we see by the multiplication rule and the continuity of the square that $R(d, \varphi)^{2}$ is defined for all values of $\varphi \neq \pi$. It remains to show that the product rule is fulfilled also for $\varphi=\pi$. Notice first that $R(d, \pi / 2)^{2}=R(d, \varphi) R(d, \pi-\varphi)=R(d, \pi)$ holds for $0<\varphi<\pi$. From this one obtains,

$$
\begin{aligned}
R(d, \pi) R(d, \varphi) & =R\left(d, \frac{\pi+\varphi}{2}\right) R\left(d, \frac{\pi-\varphi}{2}\right) R(d, \varphi)=R\left(d, \frac{\pi+\varphi}{2}\right)^{2} \\
& =R(d, \varphi-\pi) .
\end{aligned}
$$


Moreover, we get with $0<\varphi<\pi / 2$,

$$
\begin{gathered}
R(d, \pi)^{2}=R(d, \pi-\varphi) R(d, \varphi) R(d, \pi-\varphi) R(d, \varphi)=R(d, \pi-\varphi)^{2} R(d, \varphi)^{2}= \\
R(d,-2 \varphi) R(d, 2 \varphi)=1 .
\end{gathered}
$$

This implies $R(d, \pi)=R(d,-\pi)$ and the proposition is proved.

\section{References}

[BW1] Bisognano, J. and Wichmann, E.H. (1975): On the duality condition for a Hermitian scalar field. J. Math. Phys. 16, 985-1007

[BW2] Bisognano, J. and Wichmann, E.H. (1976): On the duality condition for quantum fields. J. Math. Phys. 17, 303-321

[Bch1] Borchers, H.-J. (1992): The CPT-Theorem in Two-dimensional Theories of Local Observables. Commun. Math. Phys. 143, 315-332

[Bch2] Borchers, H.-J. (1993): On Modular Inclusion and Spectrum Condition. Lett. Math. Phys. 27, 311-324

[Bch3] Borchers, H.-J. (1995): When does Lorentz Invariance imply Wedge-Duality?

Lett. Math. Phys. 35, 39-60

[BGL] Brunetti, R., Guido, D. and Longo, R. (1994): Group cohomology, modular theory and space-time symmetries. Rev. Math. Phys. 7, 57-71

[GMS] Gel'fand, I.M., Minlos, R.A. and Shapiro, Z.Ya. (1963): Representations of the rotation and Lorentz groups and their applications. Oxford, London, New York, Paris:

Pergamon Press

[GL] Guido, D. and Longo, R. (1995): An Algebraic Spin and Statistic Theorem. Commun. Math. Phys. 172, 517-533

[Ha] Haag, R. (1992): Local Quantum Physics. Berlin-Heidelberg-New York:

Springer Verlag

[Jo] Jost, R. (1965): The General Theory of Quantized Fields. Providence, RI.: Am. Math. Soc.

[Mac] Mackey, G.W. (1968): Induced Representations of Groups and Quantum Mechanics. New York, Amsterdam: W.A. Benjamin, Inc., \& Torino: Editore Boringhieri

[Wie1] Wiesbrock, H.-W. (1993): Symmetries and Half-Sided Modular Inclusions of von Neumann Algebras. Lett. Math. Phys. 28, 107-114

[Wie2] Wiesbrock, H.-W. (1993): Conformal Quantum Field Theory and Half-Sided Modular Inclusions of von Neumann Algebras. Commun. Math. Phys. 158, 537-543

\section{Communicated by G. Felder}

This article was processed by the author using the Springer-Verlag TEX PJourlg macro package 1991. 
